\title{
Range Shift in Response to Climate Change of Chinese Caterpillar Fungus Based on Species Redundancy
}

\author{
Jian Chen ${ }^{1}$, Yuan Feng ${ }^{2}$, Wu Kui ${ }^{2}$, Dai Dong ${ }^{2}$, Wang Dong ${ }^{2}$, Sun Tao ${ }^{2}$, Wang Bing ${ }^{2}$, Yang \\ Liang $^{3}$, and $\mathrm{Yu} \mathrm{Hong}^{2}$ \\ ${ }^{1}$ Yunnan Academy of Forestry and Grassland \\ ${ }^{2}$ Yunnan University \\ ${ }^{3}$ Kunming Institute of Botany Chinese Academy of Sciences
}

September 30, 2021

\begin{abstract}
The presence of the Chinese caterpillar fungus (CCF) depends on the distribution of its host insects and host plants. However, its distribution pattern in response to climate change and interspecific relationships in geographical distribution is unknown. We used the MaxEnt model to obtain areas suitable for the CCF, considering its host insects and host plants under different historical climate backgrounds. We then superimposed and analyzed them to explore the range shift in response to climate change of Chinese caterpillar fungus based on species redundancy. From the Last Glacial Maximum (LGM) to 2050, the suitable distribution pattern of the CCF is estimated to change from fragmentized to concentrated and connected. The high redundancy area (HRA) continued to increase from the Middle Holocene (MH) to the present and 2050, with an increased area of $31.46 \times 104 \mathrm{~km} 2$. The suitable area moved to the northwest and the total movement distance of its average coordinates was about $500 \mathrm{~km}$. The altitude of the suitable area increased continuously from the LGM to the present and to 2050, and the average altitude of HRA increased from $2740.89 \mathrm{~m}$ (LGM) to $4246.76 \mathrm{~m}$ (2050). The distribution pattern and changes of CCF under different climatic conditions provides a reference for the current and future geographical regional planning for conservation and sustainable utilization. The distribution pattern similarity of the CCF suitable area, suitable area for host insects, and host plants HRA of distribution area, might be the result of their long-term co-evolution. The decreasing trend of CCF yield under human disturbance was not as severe as expected, suggesting that climate change may be beneficial to distribution expansion of the CCF.
\end{abstract}

\section{Hosted file}

Range Shift in Response to Climate Change of Chinese Caterpillar Fungus Based on Species Redundancy.doc available at https://authorea.com/users/438710/articles/539822-range-shift-in-response-toclimate-change-of-chinese-caterpillar-fungus-based-on-species-redundancy 

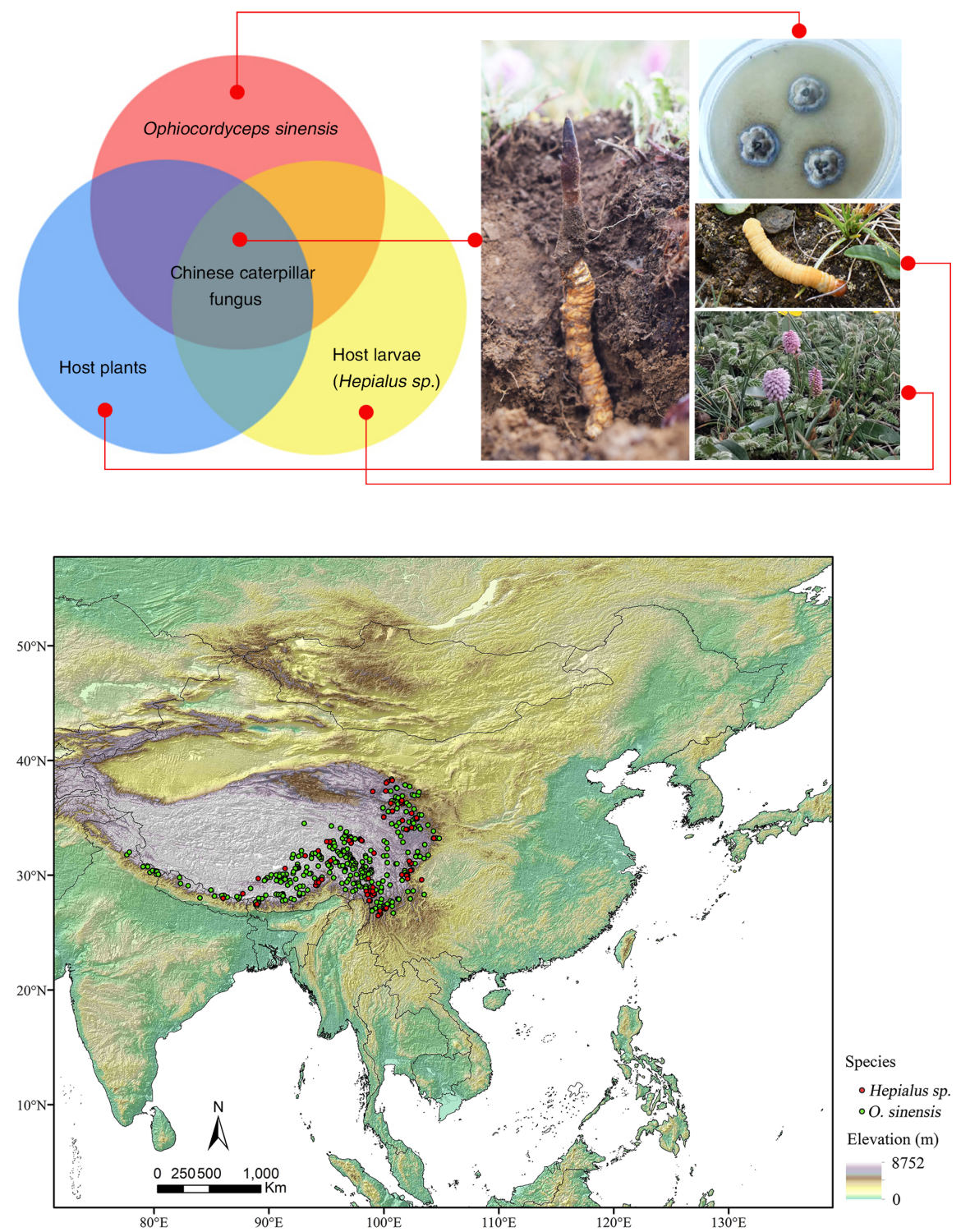

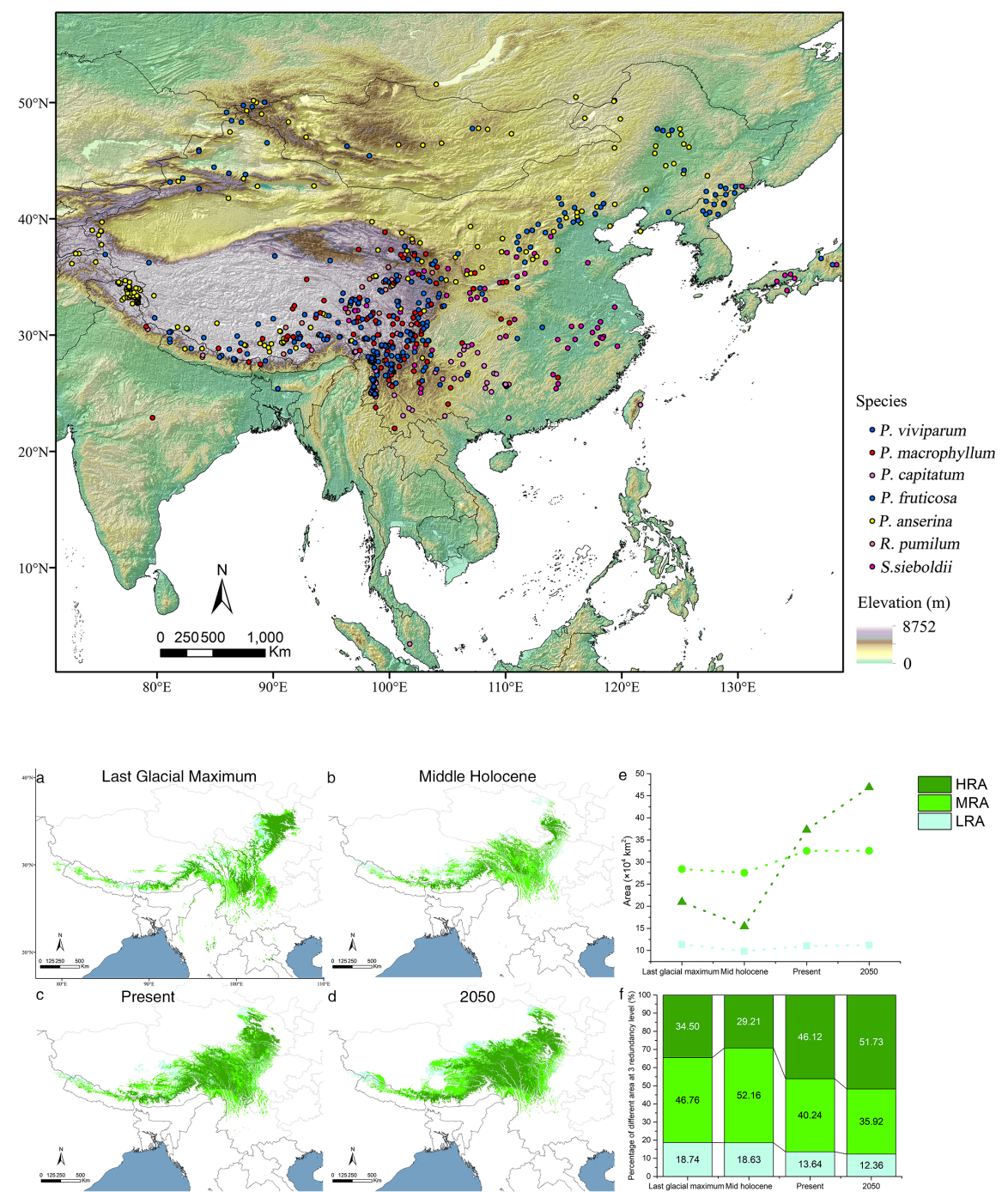

a d: Distribution pattern of 3 redundancy area in four historical periods.

e: Area change of 3 redundancy area in four historical periods.

$\mathrm{f}$ : Percentage of 3 redundancy area in four historical periods. 

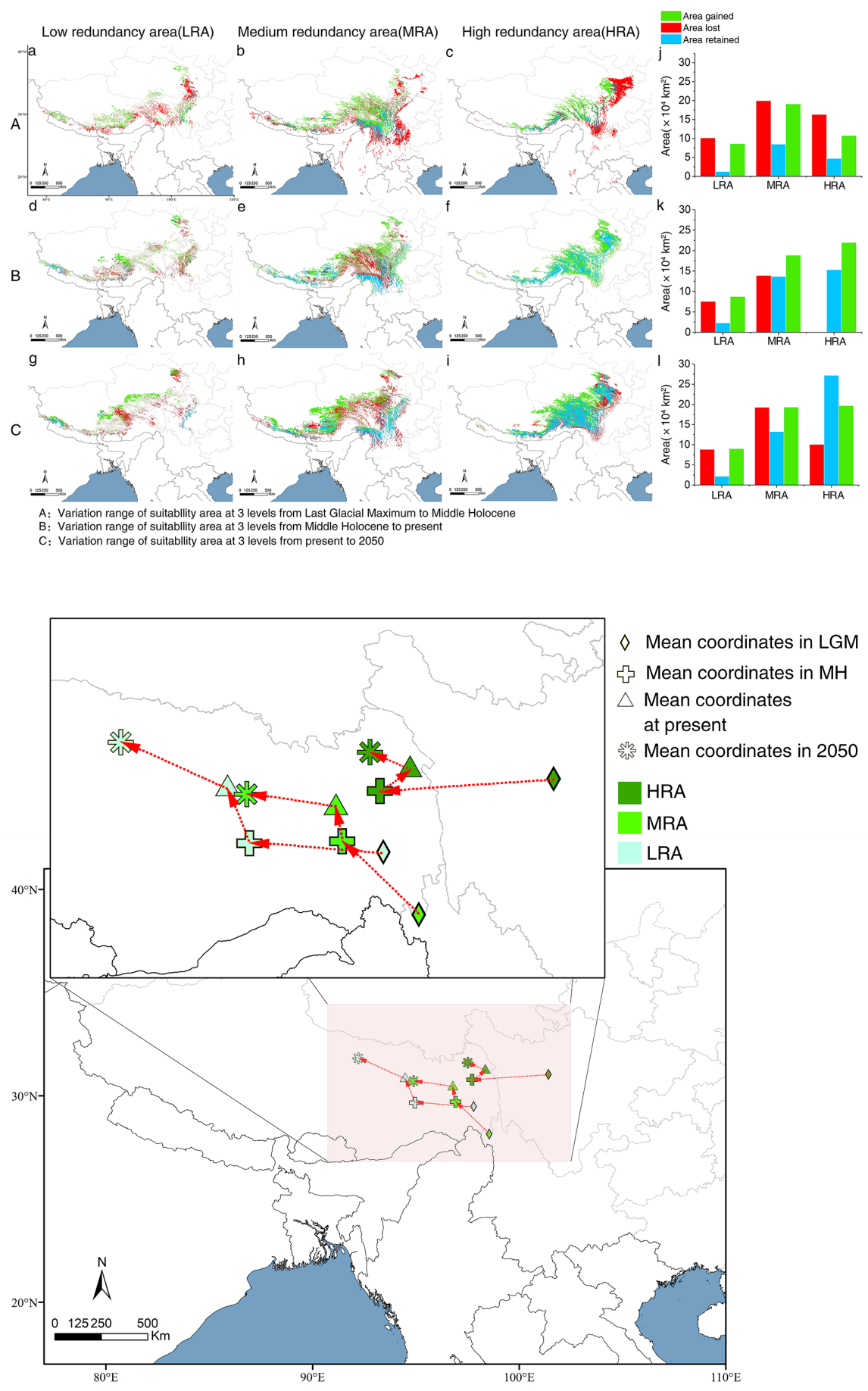

